\title{
Hebbian Mechanisms Revealed by Electrical Stimulation at Developing Rat Neuromuscular Junctions
}

\author{
Giuseppe Busetto, Mario Buffelli, Enrico Tognana, Francesco Bellico, and Alberto Cangiano \\ Dipartimento di Scienze Neurologiche e della Visione, Sezione di Fisiologia Umana, Universita' di Verona, \\ 37134 Verona, Italy
}

Synapse competition and elimination are widespread developmental processes, first demonstrated at neonatal neuromuscular junctions. Action potential activity was long shown to exert a powerful influence, but mechanisms and contribution relative to other factors are still not well understood. Here we show that replacement of natural motoneuronal discharge with synchronous activity suppresses elimination of polyneuronal innervation of myofibers. This requires the simultaneous chronic conduction block (tetrodotoxin) and distal electrical stimulation of motor axons during ectopic synaptogenesis in denervated adult soleus muscle. If in fact chronic stimulation is applied without central block of motor axons, the time course of syn- apse elimination is as fast as in control muscles undergoing natural activity. Our findings follow the prediction of Hebb's postulate and imply that asynchronous activity drives developmental synapse elimination in muscle. They further suggest that motoneurons could become transiently synchronized during development and regeneration, helping to establish the initial polyneuronal innervation.

Key words: synapse elimination; synchronous activity; synaptic competition; neuromuscular junction; polyneuronal innervation; chronic conduction block; chronic nerve electrical stimulation; development of neural connections
Synapse elimination is a basic developmental process in peripheral nervous system and CNS. At mammalian neuromuscular junctions (NMJs), the adult pattern of mononeuronal innervation is preceded by transient polyneuronal innervation during late embryonic and early postnatal life (Redfern, 1970; Bennett and Pettigrew, 1974; Brown et al., 1976; Betz et al., 1980; Jansen and Fladby, 1990). Action potential activity strongly controls this process. Synapse elimination is in fact suppressed by block of activity (Benoit and Changeux, 1975; Thompson et al., 1979; Brown et al., 1981), whereas it is accelerated by its increase induced by electrical stimulation (O'Brien et al., 1978; Thompson, 1983; Magchielse and Meeter, 1986; Nelson et al., 1993).

In neonatal visual system, procedures that establish asynchronous activation of inputs from the two eyes (strabismus) promote monocular connections on cortical neurons (Hubel and Wiesel, 1965). Normal development of binocular connections is thus interpreted as induced by synchronous firing of ocular inputs converging on these neurons (Hubel and Wiesel, 1965; Stryker and Strickland, 1984; Katz and Shatz, 1996). The general theory was proposed that synchronous activity inhibits, whereas an asynchronous one favors competition between inputs on a common target. In the visual system it is thus not activity as such that matters but temporal differences in activity in the competing inputs.

Becasue electrical stimulation evokes synchronous firing in axons, the reported acceleration of synapse elimination at the NMJ by nerve stimulation appears puzzling. One would expect stabilization of polyneuronal innervation, should the same influ-

\footnotetext{
Received June 29, 1999; revised Sept. 15, 1999; accepted Oct. 22, 1999.

This work was supported by Telethon-Italy Grant 1002 .

Correspondence should be addressed to Alberto Cangiano, Dipartimento di Scienze Neurologiche e della Visione, Sezione di Fisiologia Umana, Strada Le Grazie 8, 37134 Verona, Italy. E-mail: cangia@borgoroma.univr.it.

Copyright (C) 2000 Society for Neuroscience $0270-6474 / 00 / 200685-11 \$ 15.00 / 0$
}

ences observed in the visual pathways apply. This issue is important because if the findings on the visual system and the NMJ can be reconciled, it would provide strong support for the general theory of the role of activity on synaptic competition. Furthermore, it would strengthen the notion that mechanisms that govern synapse elimination at the NMJ hold true for central synapses as well.

Therefore, we have examined the effects of pure synchronous activity on elimination of NMJs in vivo. To obtain this, one must replace the natural discharge of motor axons with coincident activity, which requires combining their electrical stimulation with a centrally located block of conduction. Because these manipulations are practically impossible in embryos or newborn animals, we resorted to novel synapse formation in adult rats. A good model is the transplantation of a foreign nerve on a synapsefree region of soleus muscle. Section of the original nerve rapidly activates the formation of multiple inputs on each myofiber by the foreign nerve, followed by synapse elimination (Brown et al., 1976). As these junctions are new in both presynaptic and postsynaptic components, we reasoned that this paradigm would most closely mimic embryonic and neonatal synapse elimination (see Materials and Methods, also for citations), although the elimination also occurs after reinnervation of the original postsynaptic sites (Rich and Lichtman, 1989; Ribchester, 1993).

Using this model, we observed that synchronous activity suppresses synapse elimination because myofibers retain their polyneuronal innervation for at least 1 month.

Parts of this work have been published previously (Busetto et al., 1998).

\section{MATERIALS AND METHODS}

Surgical procedures and nerve transplantation. All procedures obeyed Italian laws for protection of the experimental animals. Surgery was performed under ether anesthesia on 180-350 gm male Wistar rats. The central stump of the superficial fibular nerve was transplanted onto the 
proximal surface of soleus muscle, bilaterally. Skin wounds were sutured, and rats recovered from anesthesia. After 2-3 weeks, during which foreign axons grow on a synapse-free region of soleus surface, original soleus axons were cut bilaterally (removing $\sim 1.5 \mathrm{~cm}$. of posterior tibial nerve, to prevent reinnervation), whereas tetrodotoxin (TTX) block and distal electrical stimulation started concomitantly monolaterally. Synapse formation by foreign axons begins bilaterally 3-4 d after cutting the soleus axons (Fex and Thesleff, 1967). We thus compared the effects of synchronous activity on the experimental side with those of natural activity on the control side, other factors being equal, including possible TTX systemic actions. Other rats with foreign nerve transplants but with block alone or electrical stimulation alone, were additional controls. Fibular nerve-evoked contraction was detected visually in the dissecting microscope and recorded with strain gauge in preliminary experiments. Infrequent failures $(\sim 15 \%$ in the entire study, comparable in stimulated and control) with no visible nerve implant were probably attributable to the nerve having slipped off soleus surface soon after transplantation. No reinnervation by the original nerve ever occurred. Reinnervation by the fibular nerve was quick and consistent with either active or TTX-blocked axons. The extent of reinnervation was in fact comparable in the muscles treated with the different protocols of this study, as indicated by the amplitude of their indirect isometric twitches: experimental, $4.3 \pm 0.58$ gm/100 mg muscle weight, $n=15$; control, $3.6 \pm 0.36, n=33$; TTXblocked alone, $3.8 \pm 0.64, n=12$; stimulated alone, $5.8 \pm 3.3, n=6$. Intracellular recordings showed that first layer myofibers near the fibular outgrowth (where measurements of polyneuronal innervation were made) undergo early reinnervation (day $3-5$ ).

It has been shown that adult ectopic synapse formation recapitulates embryonic synaptogenesis. Acetylcholine receptors (AChRs) accumulate at nerve-muscle contacts (Lømo and Slater, 1980a; Weinberg et al., 1981), extrajunctional AChRs disappear (Lømo and Slater, 1980a), junctional ones acquire metabolic stability (Reiness and Weinberg, 1981) and switch from $\gamma$ - to $\epsilon$-subunit (Brenner et al., 1994), acetylcholinesterase (Lømo and Slater, 1980b) and other components of basal lamina (Weinberg et al., 1981) accumulate, junctional folds develop (Korneliussen and Sommerschild, 1976) and, finally, innervation is initially polyneuronal followed by synapse elimination (Brown et al., 1976).

TTX conduction block. A special silicone cuff (9 mm length, $1.4 \mathrm{~mm}$ inner diameter, $5 \mathrm{~mm}$ outer diameter) was placed around the sciatic nerve and received a TTX solution, through a tubing connected to an Alzet miniosmotic pump (2ML4; Alza, Palo Alto, CA) implanted subcutaneously. The technique was previously shown by us to ensure complete conduction blocks, not to damage the nerve or block axonal transport or interfere with trophic functions of motor terminals (Pasino et al., 1996). TTX dose was $7.2 \mu \mathrm{g} / \mathrm{d}$.

Chronic electrical stimulation. Stimulating leads were stainless steel multistranded wires (AS-632; Cooner Wire, Chatsworth, CA) with bare tips sutured close to the common peroneal (CP) nerve, distal to the site of block. Freely moving rats were connected through tethers and electrical swivels (Chatam, Hawthorne, CA) to a stimulator. Trains of $0.1-0.3 \mathrm{msec}$ voltage pulses were varied in frequency and repetition rate in different rats. During the first $10 \mathrm{~d}$, train pulse frequency was low (15 $\mathrm{Hz}$, occasionally 30 ), then increased when planned to $80 \mathrm{~Hz}$; each train contained 8 pulses and thus varied in duration $(\sim 0.1-0.5 \mathrm{sec})$ depending on frequency. This avoided fatigue in synaptic transmission, especially at newly formed junctions, which appeared as a decline in tetanic contractions evoked by the transplanted nerve (but not by direct stimulation) that was frequency-dependent (present above $30-40 \mathrm{~Hz}$ ). After an initial period of 7-8 d after section of the original nerve and induction of synaptogenesis by the foreign nerve, this decline was no longer observed. Pulse strength was set at the onset of chronic stimulation and readjusted thereafter several times a day to insure the stimuli remained supramaximal for motor axons. To select the appropriate strength, in preliminary experiments we recorded contractions elicited by the $\mathrm{CP}$ nerve in the following muscles: extensor digitorum longus (EDL), tibialis anterior (TA), peroneus brevis (PB), and peroneus longus (PL). Thresholds were similar, i.e., within $20 \%$, whereas maximal contraction of each muscle was obtained at 1.3-1.5 $\times$ its own threshold. EDL and TA muscles were left innervated in the chronic experiment, whereas PB and PL nerves (called superficial fibular) were transplanted to reinnervate the soleus. We thus used threshold for dorsal flexion of the foot (TA and EDL, foot-threshold) as a reliable indicator of threshold for contraction of the fibular-reinnervated soleus and considered a stimulus intensity to be largely supramaximal for superficial fibular axons if it was $3 \times$ footthreshold (1.8 $\times$ being just sufficient, combining the indicated variations).

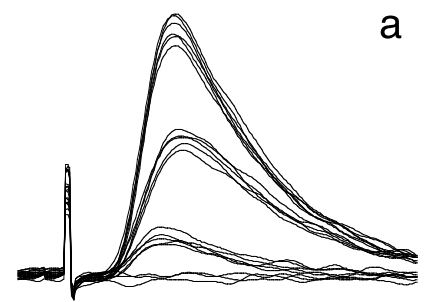

b

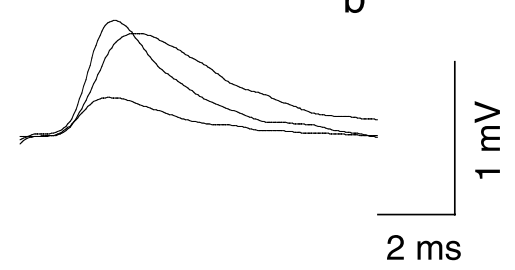

Figure 1. Example of a polyneuronally innervated fiber recorded in the region of the fibular nerve growth, showing three steps of the EPP evoked by graded stimulation of fibular nerve under curare $(a$, superimposed traces) and the components of the full-sized potential of the same fiber obtained, in isolation, by computer subtraction $(b)$ (see Materials and Methods for details).

The routine observations made on each experimental rat confirmed the adequacy of the $3 \times$ foot-threshold criterion. First, during the chronic stimulation period, foot-threshold variation between consecutive observations was small (mean, $+5 \%$ ). Second, during the final dissection in vivo and with the stimulating electrodes still in place, a clear contraction of soleus fibers reinnervated by the transplanted nerve was evoked by $\mathrm{CP}$ nerve stimulation and was maximal at the strength used in the chronic experiment. This was judged as follows: (1) threshold for visible contraction was well below the stimulation strength used in the chronic experiment and close to foot-threshold, (2) maximal contraction of fibularreinnervated soleus subsequently measured with strain gauge during the in vitro experiment, was reached at $1.3-1.5 \times$ its threshold strength. Intensities higher than $3 \times$ foot-threshold were not used to avoid stimulation of pain afferents, even though most rats had a TTX block. Unmyelinated $\mathrm{C}$ fibers evoke unbearable pain but their threshold is at least $100 \times$ that of motor fibers. Also small myelinated pain afferents (A $\delta$ component) were not excited because their range is $4.5-12 \times$ threshold for $\mathrm{A} \alpha$ motor fibers (Erlanger and Gasser, 1937). Accordingly, our stimulus strength of $3 \times$ foot-threshold never induced behavioral indications of pain, including the few unblocked rats.

Electrophysiology. Soleus muscles, after dissection, were placed in a chamber under oxygenated solution and myofibers recorded intracellularly (details in Pasino et al., 1996). No miniature endplate potentials (MEPPs) could be recorded at midbelly, because of degeneration of the cut soleus axons (exceptions were attributable to fibular reinnervation of some endplates, in a few muscles). Numerous MEPP foci were instead detected near foreign nerve outgrowth, clearly visible midway between center and proximal tendon $(\sim 2-7 \mathrm{~mm}$ from both). Under curare $(5 \times$ $10^{-7}-5 \times 10^{-6} \mathrm{~g} / \mathrm{ml}$, D-tubocurarine; Sigma, Milano, Italy), steps in endplate potentials (EPPs) were evoked by electrical stimulation of the fibular nerve by single shocks of gradually increasing intensity: fibers with two or more steps were labeled as polyneuronally innervated. Each muscle was then characterized by one number indicating the percentage of polyneuronally innervated fibers over the total number of fibers recorded in that muscle (on average $\sim 15$ fibers per muscle). Recordings were made only from first layer myofibers, and records were stored on computer and processed with Axograph. An example of a polyneuronally innervated myofiber is presented in Figure $1 a$, in which three levels of the endplate potential are obtained under curare by graded nerve stimulation, indicating that this fiber receives synaptic input by at least three different motor axons. Endplate potentials were further analyzed by characterizing their time course with a single time value which resulted from the sum of rise-time and half decay time (see ahead in Results, section on synaptic inputs). This could be done not only on the single EPP of mononeuronally innervated fibers but also on the multiple steps of polyneuronally innervated ones. To this end, each step of the composite EPP was computed by subtraction (Fig. 1b, based on the same fiber 


\begin{tabular}{|c|c|c|c|c|c|c|}
\hline Muscle treatment & $\begin{array}{l}\text { Twitch } \\
\text { duration }^{b} \\
(\mathrm{msec})\end{array}$ & $\mathrm{RMP}(\mathrm{mV})$ & $\begin{array}{l}\text { Resistance of } \\
\text { action potentials } \\
\text { to } \operatorname{TTX}^{e}(\mathrm{~V} / \mathrm{sec})\end{array}$ & $\begin{array}{l}\text { Extrajunctional } \\
\text { AChRs }^{e} \text { (femtomoles } \\
\text { of }{ }^{125} \mathrm{I}-\alpha \mathrm{Btx} / \mathrm{mg} \text { ) }\end{array}$ & F-CSA $\left(\mu \mathrm{m}^{2}\right)$ & $\begin{array}{l}\% \text { polyneuronal } \\
\text { innervation }\end{array}$ \\
\hline \multirow{2}{*}{$\begin{array}{l}\text { Nerve block }+ \\
\quad \text { distal stimulation }\end{array}$} & $97.5 \pm 7.25$ & $76.5 \pm 1.17$ & $3.7 \pm 1.00$ & $15.9 \pm 1.62^{f}$ & $2376 \pm 929 \mathrm{SD}^{g}$ & $54.6 \pm 5.91$ \\
\hline & (5) $[32-36]$ & $\begin{array}{l}(3,60)[32-36] \\
78.4 \pm 1.58^{c} \\
(3,26)\end{array}$ & $(6,83)[15]$ & (5) [15] & $(3,1763)[32-34]$ & (16) [10-34] \\
\hline \multirow{2}{*}{$\begin{array}{l}\text { Control (natural } \\
\text { impulse activity) }\end{array}$} & $96.2 \pm 8.38$ & $73.3 \pm 0.81$ & $3.2 \pm 1.42$ & $16.8 \pm 3.06^{f}$ & $2592 \pm 1001 \mathrm{SD}^{g}$ & $18.3 \pm 2.65$ \\
\hline & (11) [32-36] & $(11,213)[32-36]$ & $(4,52)[15]$ & (5) [15] & $(3,1792)[32-34]$ & (34) $[10-36]$ \\
\hline TTX-block alone & $\begin{array}{l}161 \pm 11.57 \\
(5)[32-36]\end{array}$ & $\begin{array}{l}61.9 \pm 2.4^{d} \\
(5,63)[32-36]\end{array}$ & $\begin{array}{l}76.7 \pm 4.05 \\
(2,27)\end{array}$ & $\begin{array}{l}58.1 \pm 3.48 \\
\text { (3) }[15]\end{array}$ & $\begin{array}{c}598 \pm 380 \mathrm{SD} \\
(3,7635)[32-34]\end{array}$ & $\begin{array}{l}53.6 \pm 10.01 \\
\text { (12) [10-34] }\end{array}$ \\
\hline Stimulation alone & & & & & $\begin{array}{l}2221 \pm 998 \mathrm{SD}^{g} \\
(3,2357)[32-33]\end{array}$ & $\begin{array}{l}19.9 \pm 4.01 \\
(6)[10-15]\end{array}$ \\
\hline Denervation $^{a}$ & & & $\begin{array}{l}85.5 \pm 4.88 \\
(7,25)[15]\end{array}$ & $\begin{array}{l}69.8 \pm 11.67 \\
(10)[15]\end{array}$ & & \\
\hline Normal & $\begin{array}{l}98.5 \pm 6.95 \\
(4)\end{array}$ & $\begin{array}{l}75.3 \pm 1.05 \\
(2,20)\end{array}$ & $\begin{array}{l}0 \pm 0 \\
(3,30)\end{array}$ & $\begin{array}{l}2.7 \pm 0.53 \\
(5)\end{array}$ & $\begin{array}{l}2194 \pm 656 \mathrm{SD} \\
(1,1333)\end{array}$ & \\
\hline
\end{tabular}

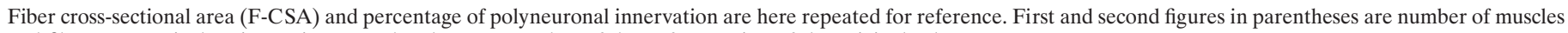
and fibers, respectively. Figures in square brackets are number of days after section of the original soleus axons.

${ }^{a}$ Denervated regions of experimental and control muscles.

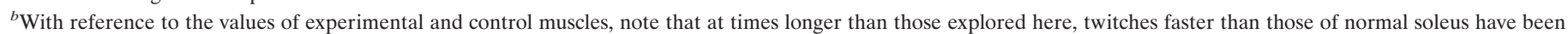

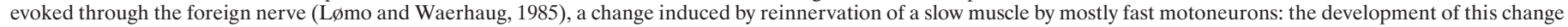

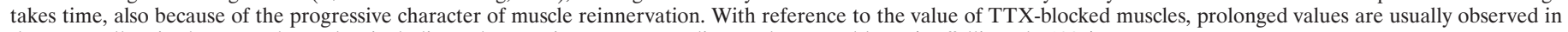
these as well as in denervated muscles, including soleus at times corresponding to those used here (Buffelli et al., 1997).

${ }^{c}$ Polyneuronally innervated fibers only.

${ }^{d}$ TTX-blocked muscles are known to have the low RMPs typical of denervated muscles (Cangiano et al., 1977).

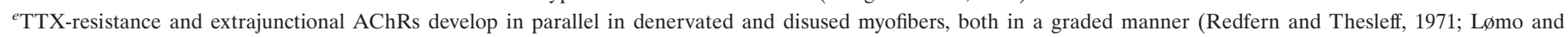

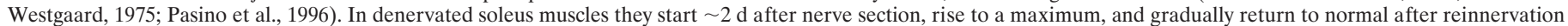

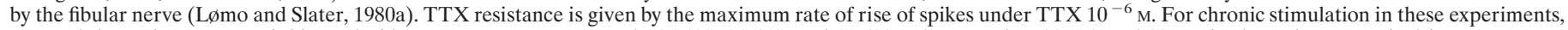
we used the main patterns of this study (three patterns, two rats each: $86,400,34,560$, and 11,520 pulses per day; 80 , 15 , and $80 \mathrm{~Hz}$ in the train, respectively).

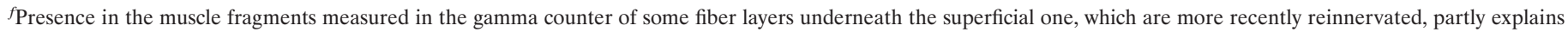
why the values, though low, have not yet reached those of normal muscles.

${ }^{g}$ Only large, putatively innervated fibers underneath the foreign nerve implant (see Fig. $5 a$ ).

and EPPs of 1a) and its time value determined independently. Resistance of action potentials to TTX was measured intracellularly with a double micropipette technique. Briefly, a recording micropipette was inserted in a given muscle fiber in the region of the foreign nerve outgrowth; a second micropipette was then inserted in the same fiber at $50-100 \mu \mathrm{m}$ of distance and used to elicit action potentials by passing short $(10 \mathrm{msec})$ depolarizing current pulses. Resistance to $10^{-6} \mathrm{M}$ TTX in the bath was signaled by the possibility of eliciting action potentials, whereas amount of resistance was given by their maximum rate of rise (volts per second) (Cangiano et al., 1984; Table 1 legend). Several fibers (10-15) were measured to characterize each muscle. Twitch and tetanic isometric contractions evoked in soleus muscles by fibular nerve stimulation were recorded in vitro soon after isolation, using a Grass FT03E force transducer (Warwick). Contractions were also measured in vivo in normal muscles: PB, PL, EDL, and soleus. All measurements were made at $26^{\circ} \mathrm{C}$.

Histology. Muscles were frozen in isopentane in liquid nitrogen, and 10 $\mu \mathrm{m}$ cross sections were prepared. To visualize the foreign nerve region, fibular axons and myofibers were stained using specific antibodies, on sections made in that region (see Fig. 4a1). Stains: axons, primary anti-neurofilament antibody (1:80), and FITC-labeled secondary antirabbit (1:160); myofibers, primary anti-dystrophin (1:400) and secondary biotin-conjugated anti-mouse (1:300), revealed with TRITC-extravidin (1:200) (Sigma). To measure fiber cross-sectional areas (F-CSA), sections made at midbelly (see Fig. 4a2) were used. Biotin was revealed with peroxidase-avidin (1:100; Dako, Milano, Italy). For visualization of synapses, longitudinal muscle sections, passing through both the surface of the foreign nerve implant and that opposite to it, were made. Axons and terminals were visualized with silver-gold impregnation and endplates with an ACh-esterase stain (Pestronk and Drachman, 1978). Sections were also used for estimating the diameter of innervated fibers up to a $300 \mu \mathrm{m}$ depth below the surface containing the foreign nerve implant.

Images of muscle cross sections at the level of nerve implant were acquired separately through a Zeiss Axiovert $35 \mathrm{~m}$ microscope and a black and white Hamamatsu video camera on an Apple G3 computer and processed with PhotoShop: axons were displayed in green, and myofibers were displayed in red and combined in one image. Images of longitudinal sections stained for synapse visualization were acquired with a Pixera (Los Gatos, CA) color video camera. Prints were made using an Epson Stylus Photo 700.

AChR labeling. Soleus muscles were stretched out and incubated with ${ }^{125} \mathrm{I}-\alpha$ BTX in oxygenated solution $\left(2 \times 10^{-8} \mathrm{M}\right.$ for $\left.1 \mathrm{hr}\right)$, washed for $2 \mathrm{hr}$, fixed overnight in glutaraldehyde $3 \%$ in $0.1 \mathrm{M} \mathrm{Na}$ cacodylate buffer, and subjected to two consecutive procedures: autoradiography of the entire muscle surface and quantitative determination of labeled sites in extrajunctional regions with gamma counter. Muscles were thinned down by surgically removing $\sim 2 / 3$ of muscle thickness, keeping the surface containing the foreign nerve intact, to eliminate deep fibers that are reinnervated later and retain extrajunctional AChRs longer than superficial fibers. Muscles were flattened with weights overnight and then placed, face down, on autoradiography film (Biomax MR; Eastman Kodak, Rochester, NY) for $4 \mathrm{~d}$. Autoradiographic images of experimental and control muscles exhibit, after $\sim 10 \mathrm{~d}$ of reinnervation, a longitudinal bundle of fibers of varying widths with very low levels of extrajunctional AChRs, corresponding to the foreign nerve implant (data not shown). This contrasts with not yet or recently reinnervated muscle that appears dark gray because of high level of extrajunctional AChRs. Using the autoradiographic images as a guide, the entire extrajunctional region was dissected out, keeping the innervated and denervated parts of each 
Figure 2. Synchronous activity, evoked by electrical stimulation, inhibits synapse elimination in newly formed ectopic neuromuscular junctions in adult rat soleus muscle. $a$, Electrophysiological comparison of the amounts of polyneuronal innervation of myofibers is made at the indicated times after bilateral section of the original nerve, which activates synaptogenesis by the transplanted fibular nerve. Experimental side (filled circles): TTX conduction block + distal stimulation of fibular axons. Control contralateral side (open circles): fibular axons undergoing natural impulse activity. Each data point represents the percentage (mean \pm SEM over several muscles) of polyneuronally innervated fibers (i.e. fibers with two or more steps of the evoked EPP, see Materials and Methods). Numbers above symbols indicate muscles. Five to thirty fibers per muscle (mean, 16.3; valid also for Fig. 3). ${ }^{*} p<$ $0.05,{ }^{*} p<0.005, t$ test. Exceptions to the above specifications: 19 of 34 control muscles are contralateral not to experimental muscles but to sides with block or stimulation alone or are unilateral preparations; they also had low values of polyneuronal innervation and were thus pooled together with the others. $b$, Comparison of the effects induced in different experimental muscles by changing the total number of stimuli per day shows that all amounts tested are effective; average time after section of soleus axons was comparable for the various amounts $(19.3,14.4,18.6 \mathrm{~d}$ for $11,520,34,560$, and 86,400 , respectively). Furthermore, the increase in synapse elimination is apparently equivalent regardless of the frequency of the stimuli in the train. The dashed line indicates mean level of polyneuronal innervation in the control muscles shown in $a$.

muscle separate. These were weighed up and counted separately (femtomoles of ${ }^{125} \mathrm{I}-\alpha \mathrm{BTX}$ per milligram of muscle weight).

Statistics. All data are expressed as mean \pm SEM except were SD is indicated. Two-tailed Student's $t$ test was used to determine statistical significance. $p<0.05$ was considered significant.

\section{RESULTS}

\section{Synchronous activity evoked by electrical stimulation inhibits synapse elimination}

In adult rats, we transplanted bilaterally the superficial fibular nerve onto the proximal surface of the soleus muscle and let it grow for 2-3 weeks, at which time we cut the original soleus axons. This resulted in the rapid formation of many new junctions by the fibular nerve (Materials and Methods). When cutting the soleus axons, we started concomitantly two chronic procedures on the experimental side: TTX conduction block and distal electrical stimulation of fibular axons. On the control side, which was often contralateral to the experimental side, we left the natural physiological firing of fibular axons unperturbed. Other preparations with foreign nerve transplants but with block alone or electrical stimulation alone served as additional controls.

Choice of chronic stimulation parameters was critically important. Strength was set to be supramaximal for fibular motor axons throughout the chronic stimulation period (Materials and Methods). Also important was the daily amount of stimuli and their frequency in the train. Concerning the amount, we used as a reference the natural activity that presumably reaches the new synapses through the transplanted fibular axons. These normally innervate fast muscles, i.e., PB and PL. In fact, most PL fibers are type II (Ranatunga and Thomas, 1990), and isometric twitch duration (time to peak + half relaxation time) of $\mathrm{PB}+\mathrm{PL}$ is much closer to fast EDL than to slow soleus (PB + PL, $52.5 \pm$ $3.77 \mathrm{msec}, n=4$; EDL, $39.7 \pm 6.17, n=3$; soleus, $98.5 \pm 6.95, n=$ $4 ; 26^{\circ} \mathrm{C}$ ). Best estimates of the activity of fibular axons can be derived from Hennig and Lømo (1985), who recorded in EDL in vivo motor unit discharge rates ranging from a few thousand (EDL type 1, 2600-11,200) up to $\sim 100,000$ impulses per day or more (EDL type 2). Thus, we varied in different rats the total number of pulses per day according to these guidelines. Frequency in the train was set, again in different rats, at 15,30 , or 80 $\mathrm{Hz}$, to test a wide range of values.

In a first series of experiments, we investigated the effects of synchronous evoked activity versus natural activity on the time course of synapse elimination. We examined muscles for the amount of polyneuronal innervation at different times after section of the original innervation, starting at $10 \mathrm{~d}$ (i.e., $\sim 1$ week after onset of synapse formation; Materials and Methods) and continuing at 15,22 , and $32-36 \mathrm{~d}$. We found that in the experimental muscles (nerve block + distal stimulation) the percentage of polyneuronally innervated fibers was much higher than in control muscles (not blocked or stimulated) at all times examined, the difference being highly statistically significant (Fig. 2a). The level of polyneuronal innervation in stimulated preparations declines after peaking at 15-20 d, but so does the level in control preparations and the difference between the two remains as large as it is initially. Figure $2 b$ illustrates the effects of changing parameters of electrical stimulation. By varying in different rats the number of stimuli per day over a sevenfold range $(11,520-$ $86,400)$, high levels of polyneuronally innervated fibers are invariably observed: a moderate increase is actually observed for 86,400 versus 11,520, although not reaching significance $(p=0.062)$. High levels of polyneuronal innervation are also observed irrespective of pulse frequency in the train $(15-80 \mathrm{~Hz})$.

We next investigated the effects on synapse elimination of conduction block of fibular axons applied in isolation and found that it also induces retention of high levels of polyneuronal innervation, in confirmation of previous findings (Thompson et al., 1979). Figure 3 actually shows that block alone produces effects quantitatively comparable to those induced by block combined with distal stimulation, over its entire time course.

Finally, we studied the effects of electrical stimulation alone on elimination of polyneuronal innervation. Figure 3 (gray columns) illustrates the remarkable finding that elimination of polyneuronal innervation is completely unaffected by this procedure, being comparable to that observed in control preparations (Fig. 2a). 


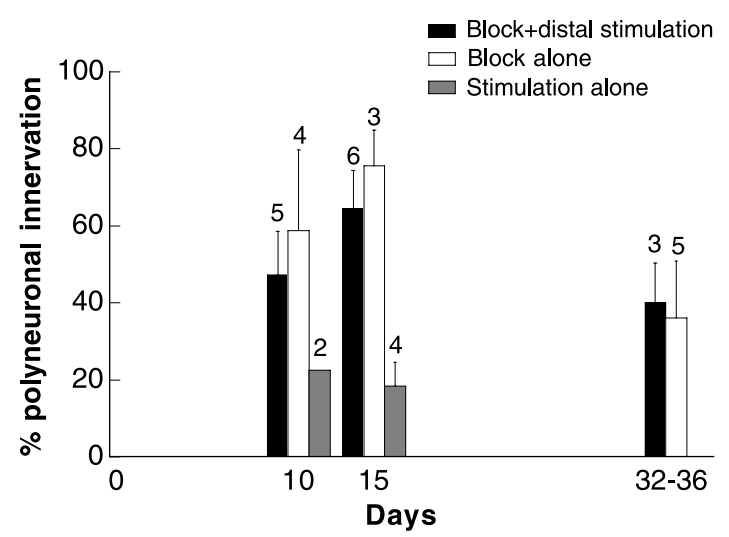

Figure 3. Comparison of the experimental muscles with similar muscles in which either stimulation or block has been omitted. Note that the muscles that were blocked but not stimulated had high levels of polyneuronal innervation. Stimulation alone, in contrast, did not prevent the elimination process. Pooling up the data at 10 and $15 \mathrm{~d}$, the difference between experimental and stimulated alone muscles is highly statistically significant $(p<0.001)$. Other indications as in Figure 2 . Note that stimulated preparations without block had a sham cuff perfusing the sciatic nerve with saline, to control for nerve damage by the blocking technique: partial denervation of fibular nerve-reinnervated myofibers would complicate the interpretation of our findings, especially in blocked muscles, because the muscle effects of partial denervation (Cangiano and Lutzemberger, 1980) could induce sprouting and enhance polyneuronal innervation. Against its occurrence is: (1) nonblocked stimulated muscles with sham cuffs actually exhibit a low percentage of polyneuronal innervation, (2) fibular-evoked twitches in preparations with cuffs are not smaller than in those without cuffs (see Materials and Methods), and (3) extensive investigations of nerves with cuffs identical to those used here, indicated lack of damage (Pasino et al., 1996).

This is at least true for the amount of stimulation used in this experiment (11,520 pulses per day), an amount that is instead able to significantly inhibit the elimination of polyneuronal innervation when combined with a TTX block central to the stimulation site (Fig. 2b).

Note that the extent of reinnervation was comparable in the muscles treated with the different approaches indicated above, as assessed through the amplitude of their indirect isometric twitches (see Materials and Methods for detailed data).

An obvious conclusion from these experiments is that even the remaining asynchronous activation present in muscles stimulated without proximal block, drives synapse elimination. However, because the levels of polyneuronal innervation seen with conduction block alone are equivalent to those obtained with conduction block and distal stimulation, we needed to know that the latter had activated the muscle fibers effectively. We show below that despite the similarity in their polyneuronal innervation, the muscles with conduction block exhibit the physiological and morphological features of inactivity, whereas the muscle fibers which were activated by the distal nerve stimulation show the features of activity present in control muscles.

\section{Electrical stimulation activates synaptic inputs as effectively as does physiological activity}

We used various independent approaches to make sure that our stimulation had been effective. First, we always found a region of large myofibers, on the side where the fibular nerve enters the muscle, whose F-CSAs are comparable on the experimental and control sides. Figure 4 illustrates three experimental muscles at different times after section of the original axons $(b)$ and one control muscle at $34 \mathrm{~d}(c)$. Note that underneath the nerve implant in the experimental muscles, only large fibers are present, just as in the control muscle. In striking contrast, much smaller fibers surround this region, on the opposite side of the foreign nerve. Small fibers are also uniformly present in soleus muscles receiving a fibular nerve transplant with conduction block alone, i.e., without stimulation $(d)$ : their small fiber cross sectional area is attributable to denervation/disuse, conditions known to induce comparable atrophy (Buffelli et al., 1997). Now, it was the population of large fibers, actually their first layer, that served in all of our experiments for microelectrode determination of polyneuronal innervation. For a quantitative comparison of experimental, control, paralyzed alone, and stimulated alone muscles, we measured fiber cross sectional areas of all fibers in the entire cross section of several muscles, at 32-34 d. The results are shown in Figure $5 a$. Both experimental and control muscles include a subpopulation ( gray columns) of large fibers comparable in number and mean fiber cross-sectional area (1763 fibers, 23.6\% of total, mean fiber cross-sectional area, $2376 \pm 929 \mu \mathrm{m}^{2} \mathrm{SD}$ for three experimental; 1792 fibers, 22.3\%, $2592 \pm 1001 \mu \mathrm{m}^{2}$ for three control muscles; difference not significant). By comparison in three TTX-blocked muscles the large fibers are absent, and the mean fiber cross-sectional area is strikingly smaller (598 \pm 380 $\mu \mathrm{m}^{2}$ ). The muscles of the "stimulation alone" condition include a population of large fibers essentially comparable (2357 fibers, $26 \%$ of total, mean fiber cross-sectional area $2221 \pm 998 \mu \mathrm{m}^{2} \mathrm{SD}$, three muscles) to those of experimental and control muscles.

The same populations of fibers of Figure $5 a$ served for another determination: we measured fiber cross-sectional areas only on the first layer myofibers under the foreign nerve. This has one advantage, focusing the attention only on that population from which microelectrode recordings of polyneuronal innervation were made. Figure $5 b$ shows comparable values in three experimental and three control muscles $\left(1882 \pm 919 \mu \mathrm{m}^{2}\right.$ mean fiber cross-sectional area, 396 fibers; $1918 \pm 1059 \mu \mathrm{m}^{2}$, 382 fibers, respectively) and a markedly smaller value in three purely TTXblocked muscles $\left(503 \pm 271 \mu \mathrm{m}^{2}, 348\right.$ fibers $)$. The stimulation alone condition again produced large superficial fibers basically similar to experimental and control muscles $\left(2114 \pm 1206 \mu \mathrm{m}^{2}\right.$, 454 fibers, three muscles).

Finally, myofiber size was also measured on longitudinal muscle sections prepared for visualization of the new synapses at $15 \mathrm{~d}$. In every section we measured the maximum apparent diameter of all fibers with multiple synaptic sites in experimental muscles and compared them with that of all innervated fibers in control muscles. Measurements were also made in a comparable number of fibers present in the same sections on the side opposite of the foreign nerve implant, devoid of neural input, markedly atrophic and considered not yet reinnervated (examples in Fig. 6a). The diameter was comparable in experimental and control muscles (respectively, $27.3 \pm 0.9 \mu \mathrm{m}, 80$ fibers; $28.9 \pm 0.9,83$; three muscles each) and much larger than in the denervated fibers (17.2 $\pm 0.6,80$ fibers, two experimental and one control muscle) (Fig. 6b).

Further evidence that our chronic stimulation was effective was obtained from functional parameters of the myofibers. For the contractile apparatus we measured the duration of fibular nerve evoked twitches. For the sarcolemma, we assessed multiple properties, either collected from each fiber in which polyneuronal innervation was determined or from similar populations of fibers 

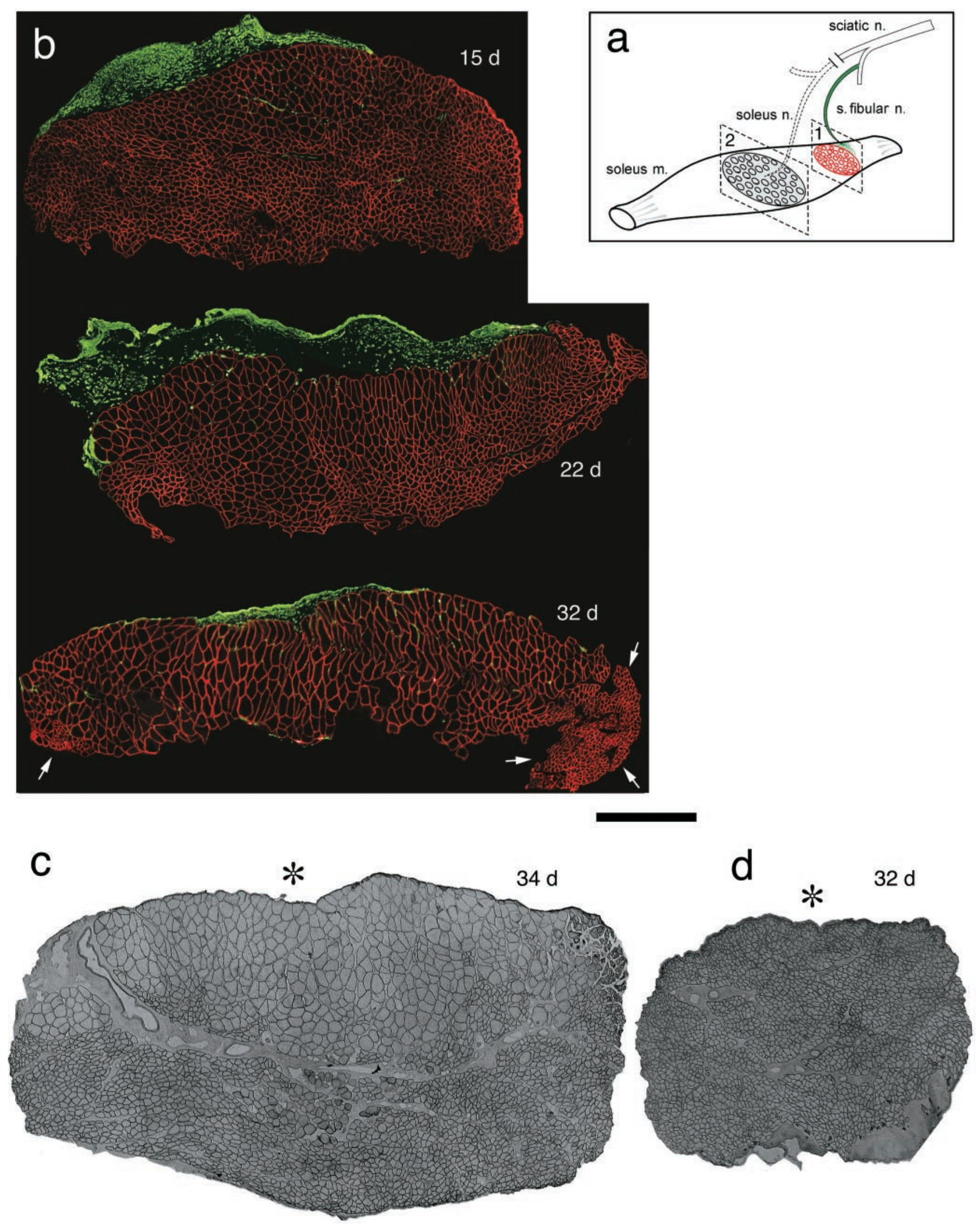

Figure 4. Cross sections of experimental $(b)$, control $(c)$, and purely paralyzed $(d)$ soleus muscles, all reinnervated by the transplanted fibular nerve, showing that in the first two conditions large fibers are present on the side of the foreign nerve outgrowth, whereas in the latter only atrophic fibers are visible. $a$, Placement of cross sections: 1 , region of fibular nerve outgrowth, used for the experimental muscles in $b$ and 2 , center of muscle belly, used for muscles in $c$ and $d$ and to collect the data shown in Figure 5. In b, muscle fibers (red) and foreign axons (green) (Figure legend continues) 
a
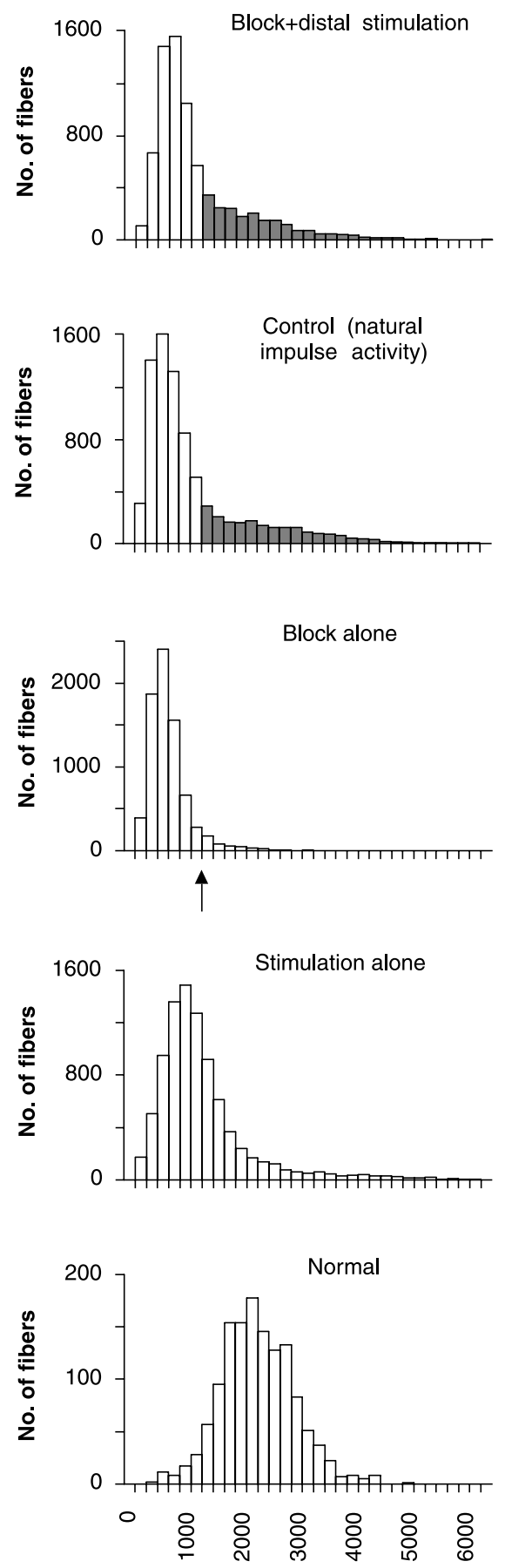

Fiber CSA $\left(\mu \mathrm{m}^{2}\right)$

\section{b Superficial fibers}
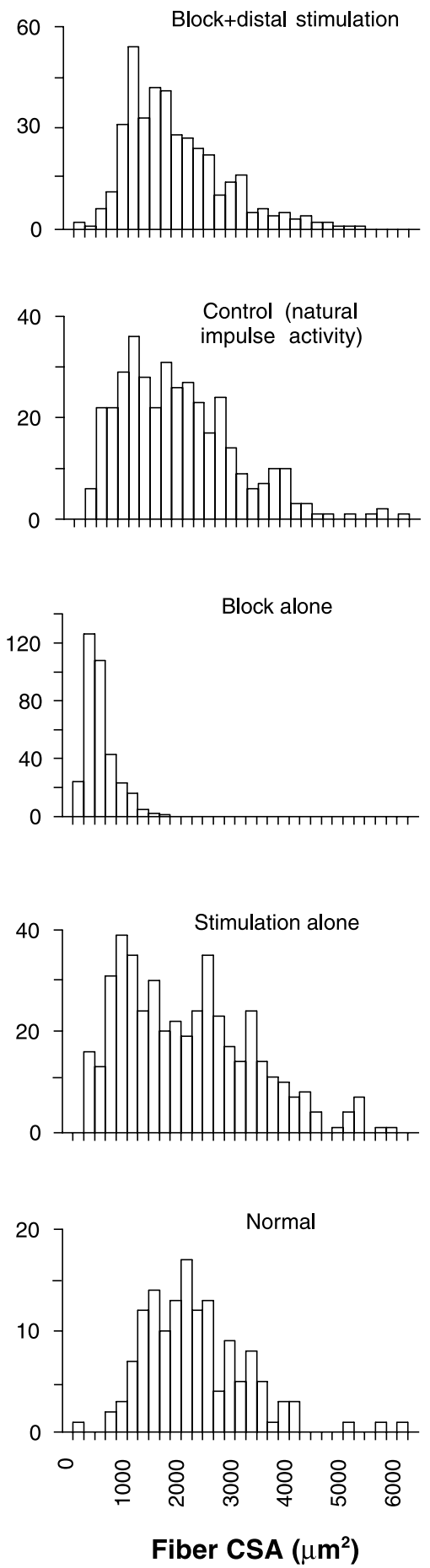

Figure 5. Comparison of frequency distribution of fiber cross-sectional areas indicates complete similarity between experimental (block + distal stimulation) and control muscles (i.e. undergoing natural activity). Only blocked, only stimulated, and normal preparations are also shown, for comparison. $a$, All fibers present in the entire transverse section through the muscle center (Fig. 4a2) of three muscles for each condition, except Normal in which only some groups of fibers are sampled from one normal soleus. $b$, Only first layer fibers of the same sections, again from three muscles for each condition (Nor$\mathrm{mal}$, one muscle), on the side of fibular nerve outgrowth. Arrow indicates a value $=$ mean $+2 \mathrm{SDs}$ for purely paralyzed muscles which is used to separate the population of large fibers in experimental and control muscles. All treatments lasting 32-34 d. Muscles of the conditions block + stimulation, control, and block alone were also used for electrophysiology, data of Figures 2 and 3; all muscles with systemic TTX, except stimulated alone ones.

are visualized with fluorescence microscopy (Materials and Methods). Stimulation parameters: $15 d, 87.5$ msec trains, 7.5 trains per minute, train pulse frequency, $80 \mathrm{~Hz}, 86,400$ pulses per day; $22 d, 467 \mathrm{msec}, 7.5$ trains per minute, $15 \mathrm{~Hz}, 86,400$ pulses per day; $32 d, 87.5 \mathrm{msec}, 1 \mathrm{train}$ per minute, $80 \mathrm{~Hz}$, 11,520 pulses per day. Two populations of myofibers are visible: (1) large fibers occupying most of the muscle cross-sectional area, in the region under nerve implant and of axonal penetration (small green spots); (2) atrophic fibers far from the nerve implant, especially tiny at margins of the muscle at $32 \mathrm{~d}$ (arrows), interpreted as not yet reinnervated fibers. Atrophic fibers are usually present on the entire side opposite to that of nerve penetration, but most of this side is not visible in the muscle at $32 \mathrm{~d}$ because it has been removed before the electrophysiological experiment, to speed up penetration of curare. $c, d$, Stain for myofibers as above, except biotin was revealed with peroxidase-avidin. *Side of implantation of fibular nerve. Scale bar, $500 \mu$ m. 


\section{a}

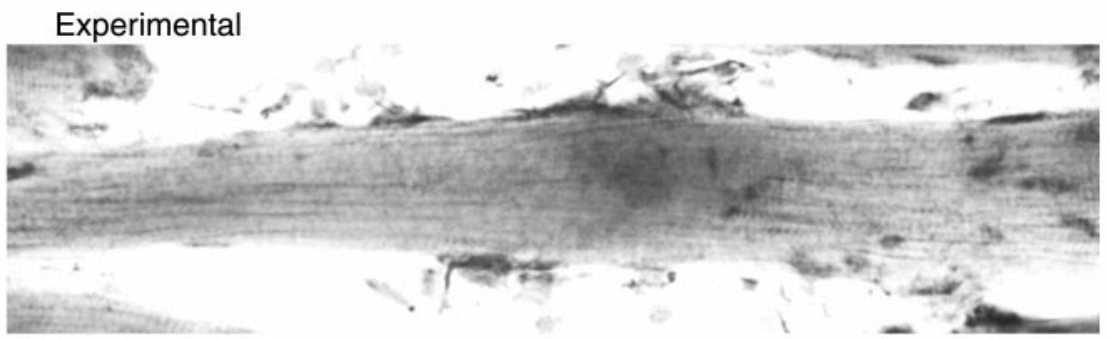

\section{Control}

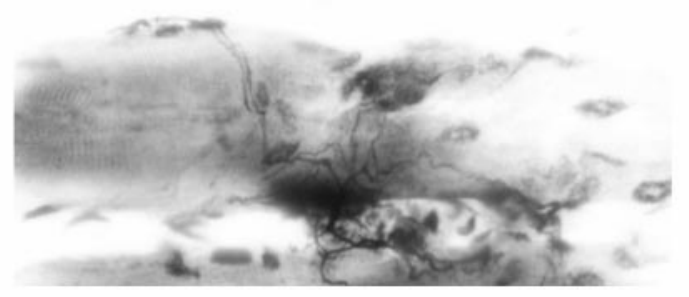

b Apparent myofiber diameter

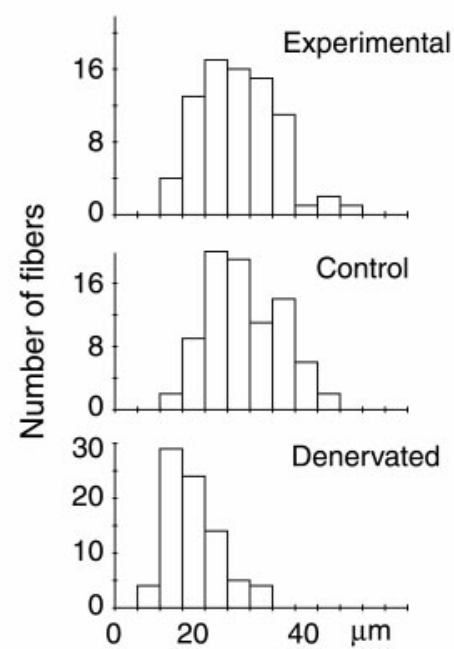

C Endplate potential duration

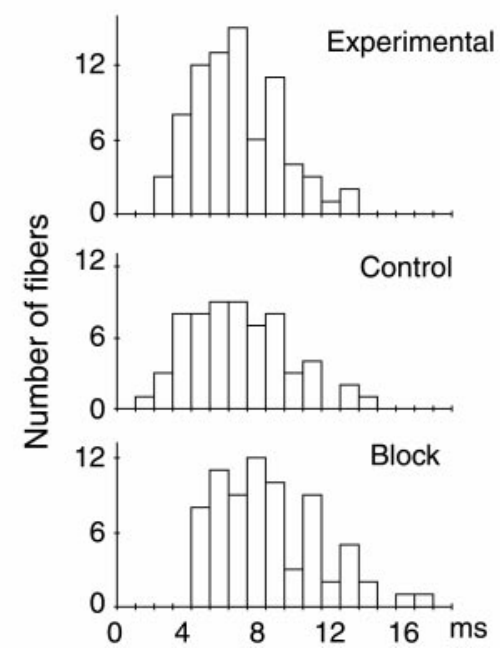

Figure 6. Morphological and functional data (EPPs) of soleus fibers undergoing multiple innervation by the transplanted fibular nerve. $a$, Longitudinal muscle sections illustrate fibers with multiple synaptic sites of experimental and control muscles at $15 \mathrm{~d}$ after section of soleus axons, as well as fibers not yet reinnervated of an experimental muscle at the same time delay. Axons and terminals are stained dark brown, and ACh-esterase are stained blue. $b$, Maximum apparent diameter measured in populations of fibers from experimental muscles (80 fibers with multiple synaptic sites), control muscles (83 fibers with single and multiple sites), and from denervated fibers of both muscle types (80 fibers, devoid of NMJs and near muscle surface opposite to fibular nerve outgrowth). $c$, Class distribution of EPP durations (rise time + half relaxation time; see Materials and Methods and Fig. 1b) at 15 and 32-36 $\mathrm{d}$, in polyneuronally innervated fibers of five experimental muscles ( 78 components, 38 fibers, $6.43 \pm 0.27$ msec, mean \pm SEM), 11 control muscles $(63$ components, 30 fibers, $6.54 \pm 0.33 \mathrm{msec}$ ) and five purely blocked muscles (73 components, 32 fibers, $8.24 \pm 0.32$ msec, significant difference with respect to both experimental and control muscles; $p<0.0005)$. Scale bar, $30 \mu \mathrm{m}$.

in dedicated experiments. To the first group belong resting membrane potential and EPP duration; to the second group resistance of action potentials to TTX and extrajunctional AChRs. The data are presented in Table 1, except EPP durations that are shown in Figure $6 c$. For all these properties the result was the same: values of experimental and control muscles were indistinguishable from each other and close to normal, whereas they both differed markedly from purely TTX-blocked muscles, which exhibited quite larger or smaller values, depending on the property examined.

\section{On distribution of synaptic inputs}

Input distribution was evaluated both morphologically and electrophysiologically. Although an exhaustive morphological study of new synapses was not performed, we examined many muscle longitudinal sections visualizing nerve endings and corresponding acetylcholinesterase accumulations in the region of the foreign nerve outgrowth. In the large majority of innervated regions of each fiber, synaptic sites were multiple in both stimulated and control muscles (average for multiply innervated regions of $3.2 \pm$ 
$0.12,126$ innervated regions, i.e. $80.3 \%$ of all regions, stimulated preparations; $3.1 \pm 0.12$, 108 regions, $77.3 \%$ of all regions control preparations) and close to each other (within a maximum of $80.0 \pm 3.85$ and $80.7 \pm 3.17 \mu \mathrm{m}$, stimulated and control preparations, respectively). Innervated regions with single synaptic sites were thus observed infrequently. In many fibers we observed more than one innervated region, the mean distance between them being $106.8 \pm 18.71 \mu \mathrm{m}$ in experimental and $193.7 \pm 20.71$ in control muscles, a highly significant difference $(p<0.005)$. Examples are shown in Figure $6 a$.

A closer comparison of the two muscle types was made using electrophysiological data. Rise time and half decay time of EPPs in polyneuronally innervated fibers were measured and added to obtain a single value characterizing the time course of each step of the composite EPP (see Materials and Methods and Fig. 1 for details). The frequency distribution of values obtained from experimental, control, and purely TTX-blocked muscles are displayed in Figure $6 c$. Although the distributions of experimental and naturally active control muscles are essentially identical, that of TTX-blocked preparations is instead shifted significantly to the right, i.e., toward a slower time course of their EPPs.

\section{DISCUSSION}

We have shown that synchronous activation of developing NMJs causes polyneuronal innervation of soleus fibers to persist longer (exploration up to 32-36 d) than in fibers whose junctions are subject to natural activity. It will be of interest to investigate even longer times and see if the stabilizing effect is permanent or slowly fades away. Polyneuronal innervation could actually outlast the stimulation period, because its persistence after a transient conduction block has been described (Barry and Ribchester, 1995). We have also shown that to observe the effects of synchronization, natural activity must be blocked, implying that its asynchronous character (Rothwell, 1994) counteracts the action of stimulation, favoring synapse competition. Moreover, we have confirmed previous findings (Thompson et al., 1979) that conduction block alone makes polyneuronal innervation to persist. We present however several independent lines of evidence demonstrating that when distal stimulation is combined with nerve conduction block, evoked activity is comparable in amount to the physiological activity in the foreign axons innervating control soleus muscles. First, the pattern of synchronous activation was in all likelihood within the normal range of peroneal motoneurons with respect to amount and frequency. Second and most important, except for the striking difference in the time course of synapse elimination, the two preparations behave similarly in all other respects, that is fiber size, contractile, and membrane properties (EPP duration, RMP values, extrajunctional AChRs, TTXresistant action potentials). Finally, the hypothesis that the level of activity administered was sufficient to maintain fiber size and other muscle parameters but insufficient to drive synapse elimination is also contradicted by our finding that a huge increase in the number of stimuli per day does not decrease the level of polyneuronal innervation (Fig. 2b). Asynchronous versus synchronous activity in the axons is therefore the only reasonable explanation for the observed difference in synapse elimination.

Alternative interpretations of our findings should be considered. First, we cannot exclude that occasionally the stimulus strength may have fallen below the supramaximal level for reinnervating motor axons, resulting in excitation of only part of the axons. This would create a kind of asynchronous activity in the converging inputs, because some would be silent when others are active. That this occurred for any significant length of time appears unlikely (see Materials and Methods). However, should that have happened, still polyneuronal innervation in experimental muscles was higher than in control muscles undergoing natural activity. Another hypothesis is that conduction block acts through some systemic effect of TTX, either on muscle or on motoneurons, but this can be discarded because the contralateral control side continues to undergo synapse elimination. Still another possibility is that the block acts by some direct retrograde effect on motoneuron cell bodies. Nevertheless, even in studies where conduction block was shown to induce changes in cell bodies, these were caused indirectly through muscle paralysis, because electrical stimulation peripheral (but not central) to the block reversed the changes (Czeh et al., 1978).

When compared with published data on the role of activity in synapse competition, our results generally agree with findings in the visual system but only partially with those in the neuromuscular system. As in the visual system, we find stabilization of inputs that participate in firing a common postsynaptic cell, a feature characteristic of "Hebbian" synapses (Hebb, 1949; see also introductory remarks). As regards agreement with data from the neuromuscular system, it was shown in Xenopus cultures that tetanic stimulation of one of two neurons innervating the same myofiber produces an immediate functional suppression of the synapse made by the other (Lo and Poo, 1991). This so-called "heterosynaptic suppression" does not occur if the two neurons are tetanized synchronously. Similar acute effects have been shown in multiply innervated fibers of adult rat muscle (Betz et al., 1989). However, the relationship of these immediate effects with the much slower developmental process of synapse elimination is not known. Our findings are also in agreement with the demonstration that one can destabilize and eliminate part of an adult NMJ (both presynaptic and postsynaptic component) if the AChRs of that part are blocked with $\alpha$-bungarotoxin (BaliceGordon and Lichtman, 1994). This suggests that "desynchronization" of different regions within the same synapse leads to elimination of the inactive region and that a similar mechanism may explain elimination of all but one nerve terminal in normal development, assuming that different terminals are asynchronously active (Jennings, 1994).

However, experiments testing the effects of differences in activity between multiple converging inputs during developmental synapse elimination gave conflicting results. Whereas Ribchester and Taxt (1983) and Ridge and Betz (1984) indicated a competitive advantage of active over inactive inputs, Callaway et al. (1989) found the opposite to be true (see also Ribchester, 1993). No explanation for the last behavior, termed anti-Hebbian (Nelson et al., 1993) and for the discrepancy between the two sets of results has been established (Jansen and Fladby, 1990); nor can we offer one here. The most direct conflict between our findings and previous data are the accelerated synapse elimination induced by nerve electrical stimulation in soleus muscles of newborn rats (O'Brien et al., 1978; Thompson, 1983) or in culture (Magchielse and Meeter, 1986; Nelson et al., 1993), particularly of a fast pattern (Thompson, 1983). Two factors, both probably operative, can explain this discrepancy. First, natural motoneuronal activity was not suppressed in these studies and thus they may be reconciled with ours, because they should be compared with our stimulated nonblocked muscles (Fig. 3). In the latter, spontaneous activity persists and appears to counteract the effects of electrical stimulation. A second explanation relates to the relative long persistence of $\mathrm{ACh}$ hypersensitivity and associated 
likely production of an "innervation factor", in neonatal soleus muscle. ACh hypersensitivity is suppressed by activity (Lømo and Westgaard, 1975) and documented by Thompson (1983) in his preparation and, as argued by him, may help explain why stimulation speeds up elimination in the newborn rat. This may also apply to similar effects of stimulation in culture (Magchielse and Meeter, 1986; Nelson et al., 1993): although not tested, ACh hypersensitivity may well be present in the myofibers because of a likely low level of spontaneous activity of innervating neurons. In our reinnervated adult soleus, ACh hypersensitivity is essentially gone by $15 \mathrm{~d}$, along with other extrajunctional membrane changes induced by denervation, and no further inhibitory effect of stimulation on this parameter and associated production of "innervation factor" can occur. The stabilizing effect of our purely synchronous activity on polyneuronal innervation can thus emerge unmasked.

In summary, our experiments provide a direct demonstration that synchronous activity of inputs during the period of synapse competition promotes the retention of polyinnervation and imply that elimination is dependent on asynchronous activity. Furthermore, they suggest that motoneurons are transiently synchronized during development and regeneration, favoring initially the formation of multiple inputs and become later desynchronized inducing elimination of redundant synapses. In favor of this possibility are reports that at least some electrical coupling between motoneurons exists early in development (Walton and Navarrete, 1991; see also Chang et al., 1998). Equally important, we show that activity has effects on neuromuscular inputs that are separate from those that it induces on muscle membrane and contractile properties, including the downregulation of an "innervation factor." Finally, our findings establish a continuity of interpretation between the neuromuscular system, in which elimination was first shown, and the visual system, in which many of the developmental paradigms related to synaptic competition and elimination were developed. Other factors may participate to the control of synapse elimination either activity-dependent or independent (Barry and Ribchester, 1995; Nguyen and Lichtman, 1996; Nguyen et al., 1998). Also, Jansen and coworkers (Thompson et al., 1979) have provided evidence for a natural tendency of motoneurons to withdraw part of their branches after the initial period of wide fields of innervation during development. In any event, the accessibility of the NMJ makes the present preparation attractive for studying synapse competition and the mechanisms of its dependence on activity pattern, at the cellular and molecular level, as well as the possible participation of other factors.

\section{REFERENCES}

Balice-Gordon RJ, Lichtman JW (1994) Long-term synapse loss induced by focal blockade of postsynaptic receptors. Nature 372:519-524.

Barry JA, Ribchester RR (1995) Persistent polyneuronal innervation in partially denervated rat muscle after reinnervation and recovery from prolonged nerve conduction block. J Neurosci 15:6327-6339.

Bennett MR, Pettigrew AG (1974) The formation of synapses in striated muscle during development. J Physiol (Lond) 241:515-545.

Benoit P, Changeux JP (1975) Consequences of tenotomy on the evolution of multiinnervation in developing rat soleus muscle. Brain Res 99:354-358.

Betz WJ, Caldwell JH, Ribchester RR (1980) The effects of partial denervation at birth on the development of muscle fibres and motor units in rat lumbrical muscle. J Physiol (Lond) 303:265-279.

Betz WJ, Chua M, Ridge RM (1989) Inhibitory interactions between motoneurone terminals in neonatal rat lumbrical muscle. J Physiol (Lond) 418:25-51.

Brenner HR, Rotzler S, Kues WA, Witzemann V, Sakmann B (1994) Nerve-dependent induction of AChR epsilon-subunit gene expression in muscle is independent of state of differentiation. Dev Biol 165:527-536.

Brown MC, Jansen JKS, Van Essen DC (1976) Polyneuronal innervation of skeletal muscle in new-born rats and its elimination during maturation. J Physiol (Lond) 261:387-422.

Brown MC, Holland RL, Hopkins WG (1981) Restoration of focal multiple innervation in rat muscles by transmission block during a critical stage of development. J Physiol (Lond) 318:355-364.

Buffelli M, Pasino E, Cangiano A (1997) Paralysis of rat skeletal muscle equally affects contractile properties as does permanent denervation. J Muscle Res Cell Motil 18:683-695.

Busetto G, Buffelli M, Tognana E, Bellico F, Cangiano A (1998) Synchronous nerve evoked activity in newly established rat neuromuscular synapses inhibits elimination of multiple inputs. Soc Neurosci Abstr 24:273.

Callaway EM, Soha JM, Van Essen DC (1989) Differential loss of neuromuscular connections according to activity level and spinal position of neonatal rabbit soleus motor neurons. J Neurosci 9:1806-1824.

Cangiano A, Lutzemberger L (1980) Partial denervation in inactive muscle affects innervated and denervated fibres equally. Nature 285:233-235.

Cangiano A, Lutzemberger L, Nicotra L (1977) Non-equivalence of impulse blockade and denervation in the production of membrane changes in rat skeletal muscle. J Physiol (Lond) 273:691-706.

Cangiano A, Magherini PC, Pasino E, Pellegrino M, Risaliti R (1984) Interaction of inactivity and nerve breakdown products in the origin of acute denervation changes in rat skeletal muscle. J Physiol (Lond) 355:345-365.

Chang O, Gonzalez M, Pinter M, Balice-Gordon R (1998) Transient gap junction mediated coupling among developing motor neurons. Soc Neurosci Abstr 24:1040.

Czeh G, Gallego R, Kudo N, Kuno M (1978) Evidence for the maintenance of motoneurone properties by muscle activity. J Physiol (Lond) 281:239-252.

Erlanger J, Gasser H (1937) Electrical signs of nervous activity. Philadelphia: University of Pennsylvania.

Fex S, Thesleff S (1967) The time required for innervation of denervated muscles by nerve implants. Life Sci 6:635-639.

Hebb DO (1949) The organization of behavior. New York: Wiley.

Hennig R, Lømo T (1985) Firing patterns of motor units in normal rats. Nature 314:164-166.

Hubel DH, Wiesel TN (1965) Binocular interactions in striate cortex of kittens reared with artificial squint. J Neurophysiol 28:1041-1059.

Jansen JKS, Fladby T (1990) The perinatal reorganization of the innervation of skeletal muscle in mammals. Prog Neurobiol 34:39-90.

Jennings C (1994) Death of a synapse. Nature 372:498-499.

Katz LC, Shatz CJ (1996) Synaptic activity and the construction of cortical circuits. Science 274:1133-1138.

Korneliussen H, Sommerschild H (1976) Ultrastructure of the new neuromuscular junction formed during reinnervation of rat soleus muscle by a "foreign" nerve. Cell Tissue Res 167:439-452.

Lo YJ, Poo MM (1991) Activity-dependent synaptic competition in vitro: heterosynaptic suppression of developing synapses. Science 254:1019-1022.

Lømo T, Slater C (1980a) Acethylcholine sensitivity of developing ectopic nerve-muscle junctions in adult rat soleus muscles. J Physiol (Lond) 303:173-189.

Lømo T, Slater C (1980b) Control of junctional acetylcholinesterase by neural and muscular influences in the rat. $\mathrm{J}$ Physiol (Lond) 303:191-202.

Lømo T, Waerhaug O (1985) Motor endplates in fast and slow muscles of the rat: what determines their differences? J Physiol (Paris) 80: 290-297.

Lømo T, Westgaard R (1975) Further studies on the control of ACh sensitivity by muscle activity in the rat. J Physiol (Lond) 252:603-626.

Magchielse T, Meeter E (1986) The effect of neuronal activity on the competitive elimination of neuromuscular junctions in tissue culture. Dev Brain Res 25:211-220.

Nelson PG, Fields RD, Yu C, Liu Y (1993) Synapses elimination from the mouse neuromuscular junction in vitro: a non-Hebbian activitydependent process. J Neurobiol 24:1517-1530.

Nguyen QT, Lichtman JW (1996) Mechanism of synapse disassembly at the developing neuromuscular junction. Curr Opin Neurobiol 6:104-112.

Nguyen QT, Parsadanian AS, Snider WD, Lichtman JW (1998) Hyper- 
innervation of neuromuscular junctions caused by GDNF overexpression in muscle. Science 279:1725-1729.

O'Brien RAD, Ostberg AC, Vrbová G (1978) Observation on the elimination of polyneuronal innervation in developing mammalian skeletal muscle. J Physiol (Lond) 282:571-582.

Pasino E, Buffelli M, Arancio O, Busetto G, Salviati A, Cangiano A (1996) Effects of long-term conduction block on membrane properties of reinnervated and normally innervated rat skeletal muscle. J Physiol (Lond) 497.2:457-472.

Pestronk A, Drachman DB (1978) A new stain for quantitative measurement of sprouting at neuromuscular junctions. Muscle Nerve 1:70-74.

Ranatunga KW, Thomas PE (1990) Correlation between shortening velocity, force velocity relation and histochemical fibre type composition in rat muscle. J Muscle Res Cell Motil 11:240-250.

Redfern PA (1970) Neuromuscular transmission in new-born rats. J Physiol (Lond) 209:701-709.

Redfern PA, Thesleff S (1971) Action potential generation in denervated rat skeletal muscle. II. The action of tetrodotoxin. Acta Physiol Scand 82:70-78.

Reiness C, Weinberg C (1981) Metabolic stabilization of acetylcholine receptor at newly formed neuromuscular junction in the rat. Dev Biol 84:247-254.

Ribchester RR (1993) Coexistence and elimination of convergent motor nerve terminals in reinnervated and paralysed adult rat skeletal muscle. J Physiol (Lond) 466:421-441.
Ribchester RR, Taxt T (1983) Motor unit size and synaptic competition in rat lumbrical muscles reinnervated by active and inactive motor axons. J Physiol (Lond) 344:89-111.

Rich MM, Lichtman JW (1989) In vivo visualization of pre- and postsynaptic changes during synapse elimination in reinnervated mouse muscle. J Neurosci 9:1781-1805.

Ridge RM, Betz WJ (1984) The effect of selective, chronic stimulation on motor unit size in developing rat muscle. J Neurosci 4:2614-2620.

Rothwell J (1994) Control of human voluntary movement. London: Chapman \& Hall.

Stryker MP, Strickland SL (1984) Physiological segregation of ocular dominance columns depends on the pattern of afferent electrical activity. Invest Ophthalmol Vis Sci [Suppl] 25:278.

Thompson WJ (1983) Synapse elimination in neonatal rat muscle is sensitive to the pattern of muscle use. Nature 302:614-616.

Thompson WJ, Kuffler DP, Jansen JKS (1979) The effect of prolonged reversible block of nerve impulses on the elimination of polyneuronal innervation of new-born rat skeletal muscle. Neuroscience 4:271-281.

Walton KD, Navarrete R (1991) Postnatal changes in motoneurone electrotonic coupling studied in the in vitro rat lumbar spinal cord. J Physiol (Lond) 433:283-305.

Weinberg C, Sanes J, Hall Z (1981) Formation of neuromuscular junctions in adult rats: accumulation of acetylcholine receptors, acetylcholinesterase and components of synaptic basal lamina. Dev Biol 84:255-266. 